\title{
Submillimetre Continuum Imaging of Large-scale Outflows in Nearby Starburst Galaxies
}

\author{
L.L. Leeuw and E.I. Robson \\ Centre for Astrophysics, University of Central Lancashire, Preston, UK \\ Joint Astronomy Centre, 660 N. A'ohoku Place, Hilo, HI 96720, USA \\ D.H. Hughes
}

Royal Observatory, Blackford Hill, Edinburgh, EH9 3HJ, UK

\begin{abstract}
.
We present SCUBA imaging observations of the nearby prototype starburst galaxy M82, in order to study large-scale outflows observed in such galaxies and associated with starburst phenomena. We use (1) deep $450 \mu \mathrm{m}$ continuum maps to investigate the structure and details of the large-scale outflows and (2) $850 \mu \mathrm{m}$ linear-polarization maps to investigate the relation between nuclei and halo magnetic fields.
\end{abstract}

\section{Introduction}

Massive ejections of gas and dust originating in galactic nuclei have been observed at scales of a few kpc in optical emission lines, submillimetre molecular lines, radio continuum emission and soft X-rays (Shopbell \& Bland-Hawthorn 1998, Seaquist, Frayer \& Bell 1998 and references therein). One explanation for the outflows is that a high supernova rate in the galactic nucleus heats up the surrounding gas to temperatures and sound speeds exceeding the velocity of the galaxy, creating a wind that expands outward from the galaxy (Chevalier $\&$ Clegg 1985). The outflows are usually oriented along the minor axes of the galaxies and are thus observed in edge-on galaxies.

At a distance of $3.63 \mathrm{Mpc}$, the edge-on classical starburst galaxy, M82, has dimensions of $11.2^{\prime} \times 4.3^{\prime}$, and thus provides a favourable opportunity to investigate the physical association between the galactic nuclei and the outflows, as a prerequisite to studying similar phenomena in other nearby galaxies. The results presented here are preliminary (Leeuw et al. in preparation).

\section{Observations}

We have obtained deep $450 \mu \mathrm{m}$ imaging observations of the central $<60^{\prime \prime} \times 60^{\prime \prime}$ $(1 \mathrm{kpc} \times 1 \mathrm{kpc}$ diameter $)$ and extended halo $\sim 180^{\prime \prime} \times 180^{\prime \prime}(3 \mathrm{kpc} \times 3 \mathrm{kpc})$ emission in M82. For the central $\sim 40^{\prime \prime} \times 40^{\prime \prime}(0.7 \mathrm{kpc} \times 0.7 \mathrm{kpc})$, we also have linear-polarization images at $850 \mu \mathrm{m}$, which we believe are the first extragalactic 


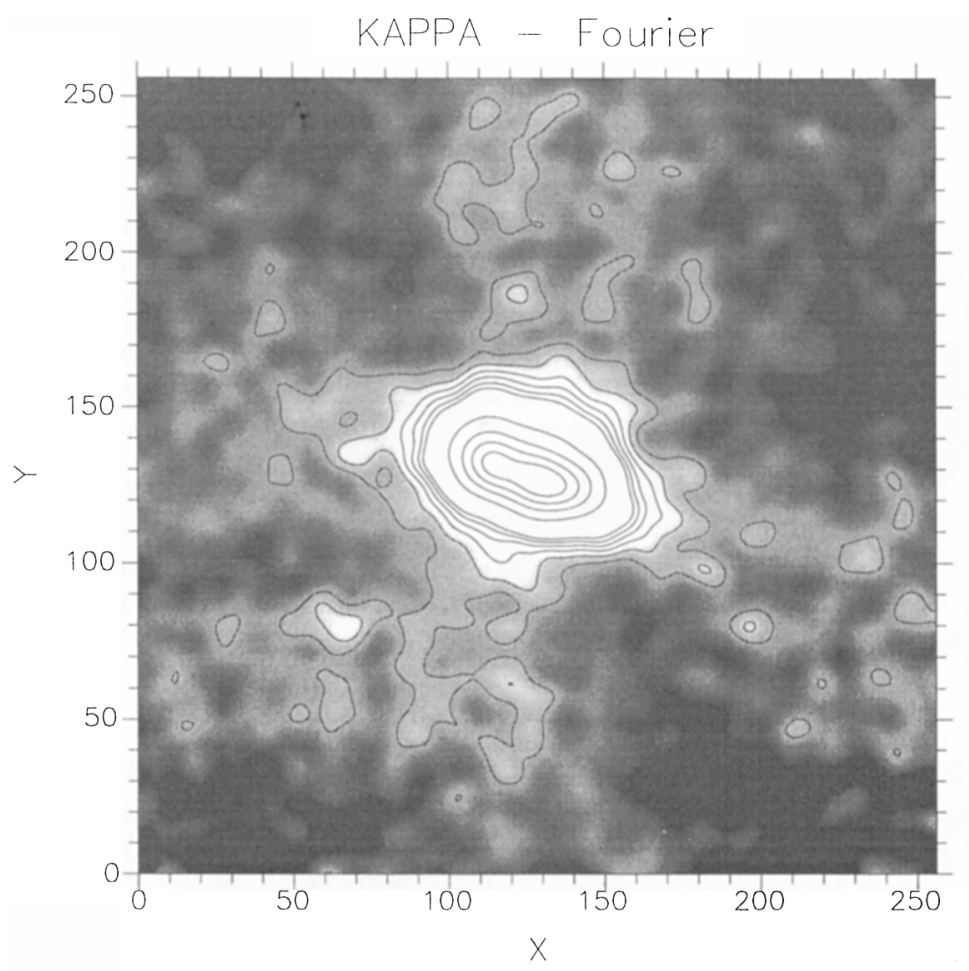

Figure 1. A deep map of the $450 \mu \mathrm{m}$ continuum emission centered at the near-infrared nucleus of M82. The X and Y axes are offsets in arcseconds. The map has been smoothed to $11^{\prime \prime}$. The rms uncertainty on the map is about $130 \mathrm{mJy} / \mathrm{beam}$. The deep $850 \mu \mathrm{m}$ map is still being reduced and is not presented here.

sub-mm polarization observations. The data were obtained with the JCMT's new submillimetre continuum camera SCUBA (Holland et al. 1998).

\section{The Large-Scale Outflows}

The high resolution maps show the double peaks $(<1 \mathrm{kpc}$ in diameter) that have been seen in the mid-infrared and CO line transitions observations, as well as in $450 \mu \mathrm{m}$ maps made with the previous JCMT bolometer, and which were modelled as thermal re-radiation from a dusty-molecular torus at about $48 \mathrm{~K}$ (Hughes et al. 1994). For the first time an asymmetrical submillimetre halo of at least $2.5 \mathrm{kpc}$ diameter is seen outside the torus (see Fig. 1). In the south the halo has two filamentary structures, each apparently associated with a separate peak of the dusty-molecular torus, and co-spatial with $\mathrm{H} \alpha$ driven winds in M82 (Shopbell \& Bland-Hawthorn 1998). This preliminary evidence 


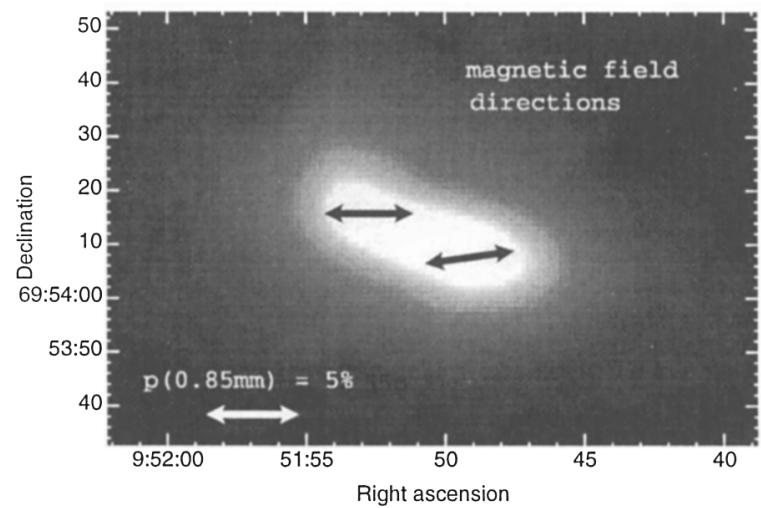

Figure 2. The $850 \mu \mathrm{m}$ map of M82 with the B-field (solid arrows) superimposed (Greaves et al. in preparation). The polarization vectors are perpendicular to the plotted vectors and the average B-field is inclined about 10 degrees parallel to the disk of the galaxy.

in terms of the filamentary structures and nuclear and halo magnetic fields (see section 4) indicates that the submillimetre halo can be interpreted as outflows of molecular-dust that is entrained in starburst-driven winds (Leeuw et al. in preparation).

\section{The Nuclear Linear-Polarized $850 \mu \mathrm{m}$ Map}

At $850 \mu \mathrm{m}$ we observe a magnetic (B) field that has two main components, each centered on a separate peak of the dusty torus and with slightly different orientation from another (see Fig. 2). The average B-field direction is inclined about 10 degrees to the disk of the galaxy. This toroidal B-field is consistent with polarization observations of other nearby disk galaxies and is complementary to the poloidal B-field that has been observed in the halo of M82 (Reuter et al. 1994) and other edge-on disks. The poloidal B-field was believed to be nuclear magnetic fields that have been driven vertically by the violent star formation in the centre of the galaxy (and in some cases ejected by forces associated with a central AGN). Reuter et al. 1994 did not observe any radio polarized emission in the nucleus of M82 because the dusty molecular torus of M82 depolarizes this radio synchrotron radiation. At submillimetre wavebands synchrotron radiation is negligible and the polarized radiation is due to dust grains aligned by a B-field. Furthermore, Faraday rotation is negligible, so any observed B-fields must be intrinsic to the galaxy. In the context of the poloidal halo B-fields observed by Reuters et al. 1994, our results provide further evidence of the physical 
association of starburst nuclei and large-scale outflows. Deeper polarization observations of M82 at both 450 and $850 \mu \mathrm{m}$ will provide further details to this picture (Greaves et al. in preparation).

\section{Remarks}

If the submillimetre large-scale detections are of dust entrained in supernovae driven-winds, this dust will have been metal-enriched and therefore the outflows will affect the evolution of the galaxy. Further work is needed to determine the velocity and rate of the expelled dust in order to constrain models of the starburst-driven outflows and their possible effect on the galaxy evolution.

Acknowledgements. Observations presented used the JCMT which is operated by the Joint Astronomy Centre, on behalf of the UK Particle Physics and Astronomy Research Council, the Netherlands Organization for Scientific Research and the Canadian National Research Council. LLL acknowledges the University of Central Lancashire for a full-time research studentship.

\section{References}

Chevalier, R.A., Clegg, A.W. 1985, Nature, 317, 44

Holland, W.H., et al. 1998, MNRAS, in press

Hughes, D.H., Gear, W.K., Robson, E.I. 1994, MNRAS, 270, 641

Reuter, H.P., Klein, U., Lesch, H., Wielebinski, R., Kronberg, P.P. 1994, A\&A, 282,724

Seaquist, E.R., Frayer, D.T., Bell, M.B. 1998, astro-ph/9806295

Shopbell, P.L., Bland-Hawthorn, J. 1998, ApJ, 493, 129

\section{Discussion}

Tolstoy: Does the gas escape from M82?

Leeuw: With our $450 \mu \mathrm{m}$ continuum emission we do not have velocity information, so we cannot say with certainty. But observations of $\mathrm{CO}$ and $\mathrm{H} \alpha$, to list just a few, have shown that the gas escapes. We are saying that if the $450 \mu \mathrm{m}$ emission is coupled to the $\mathrm{CO}$ or $\mathrm{H} \alpha$ then the $450 \mu$ emission (dust) must also escape for M82! 